Philosophie ANTIQUE

\section{Philosophie antique}

Problèmes, Renaissances, Usages

15 | 2015

Questions sur le scepticisme pyrrhonien

\title{
Tonneau percé, tonneau habité
}

Calliclès et Diogène : les leçons rivales de la nature

\section{Simon-Pierre Chevarie-Cossette}

\section{(2) OpenEdition}

\section{Journals}

Édition électronique

URL : https://journals.openedition.org/philosant/386

DOI : 10.4000/philosant.386

ISSN : 2648-2789

Éditeur

Éditions Vrin

\section{Édition imprimée}

Date de publication : 24 novembre 2015

Pagination : 149-178

ISBN : 978-2-7574-1141-4

ISSN : $1634-4561$

Référence électronique

Simon-Pierre Chevarie-Cossette, «Tonneau percé, tonneau habité », Philosophie antique [En ligne], 15 |

2015, mis en ligne le 01 novembre 2018, consulté le 02 décembre 2022. URL : http://

journals.openedition.org/philosant/386 ; DOI : https://doi.org/10.4000/philosant.386

\section{(c) $)(9)$}

Creative Commons - Attribution - Pas d'Utilisation Commerciale - Pas de Modification 4.0 International - CC BY-NC-ND 4.0

https://creativecommons.org/licenses/by-nc-nd/4.0/ 


\author{
TONNEAU PERCÉ, TONNEAU HABITÉ \\ Calliclès et Diogène : les leçons rivales de la nature \\ Simon-Pierre CHEVARIE-COSSETTE \\ Université de Montréal
}

RÉSUMÉ. Comme de nombreux penseurs antiques avant et après eux et contrairement à Socrate, Calliclès et Diogène ont déclaré avoir fondé leur éthique sur l'observation de la nature. Et pourtant, les deux discours normatifs qui sont tirés d'une nature que l'on pourrait a priori croire être la même sont on ne peut plus opposés. Calliclès croit que l'homme est appelé à dominer autrui ; Diogène pense plutôt qu'il doit se dominer lui-même; le premier est un hédoniste débridé, le second croit que le bonheur ne s'achète qu'au prix du sacrifice des désirs artificiels. Comment expliquer cette dichotomie? Nous empruntons deux routes explicatives. D'abord, nous montrons que pour Calliclès et Diogène, la notion de nature non seulement diffère, mais est observée sous un angle différent. La première est celle des tyrans et de dieux anthropomorphes, la seconde, celle de petits animaux et de dieux autarciques. La première concerne la relation des hommes entre eux ; la seconde, celle de l'homme avec lui-même. Ensuite, nous exposons la différence des présupposés normatifs qui précèdent ou accompagnent l'observation de la nature ; nous contrastons, pour ce faire, les formes d'anticonventionnalisme et d'anti-intellectualisme défendues par Calliclès et Diogène.

SUMMARY. Like many ancient thinkers before and after them and contra Socrates, Callicles and Diogenes said they based their ethics on the observation of nature. And yet, the two normative discourses that are derived from nature that we might a priori believe to be the same could not be more opposed. Callicles believes that people are called to dominate others, Diogenes rather thinks people must dominate themselves. The first is an unbridled hedonist, the second believes that happiness can be bought only at the price of sacrificing artificial desires. How to explain this dichotomy? We take two explanatory routes. First, we show that for Callicles and Diogenes, the notion of nature not only differs but is observed from a different angle. The first one is the nature of tyrants and anthropomorphic gods, the second that of small animals and autarkic gods. The first focuses on the relationship between people, the second focuses on the relationship of a person with themselves. Secondly, we expose the difference in normative assumptions that precede or accompany the observation of nature; in order 
to do so, we contrast anticonventionalism and anti-intellectualism forms proper to both Callicles and Diogenes. 
Depuis les présocratiques jusqu'aux philosophes hellénistiques, il est une constante «métaéthique $»^{1}$ qui semble avoir traversé la philosophie antique : l'appel de nombreux philosophes à la nature pour fonder la morale $^{2}$. La solidarité de l'éthique et de la physique dans la division des philosophies épicurienne et stoïcienne $e^{3}$ de même que l'attention soutenue que portèrent les présocratiques à la question de l'arche du monde, sont déjà d'excellents indices que les Grecs comprenaient la façon dont ils devaient agir à l'aune de la place qu'ils occupaient dans le cosmos. Mais ce ne saurait être vrai de tous les Grecs, car Socrate, dit-on :

$[\ldots]$ ne discutait pas [...] de la nature de toutes choses, comme le faisaient la plupart des autres, en examinant ce qu'il en est de ce que les sophistes appellent le « monde » [...] il faisait même la démonstration que ceux qui réfléchissent à de tels sujets ont perdu la raison [...]. [C]'est toujours d'affaires humaines qu'il s'entretenait, examinant en quoi consistent le pieux et l'impie, le beau et le laid, le juste et l'injuste, la modération et la folie, le courage et la lâcheté, la cité et le politicien, le gouvernement des hommes et l'aptitude à les gouverner. (Mémorables, I 1, 11-16, trad. Dorion.)

Des divergences fondamentales avec certains de ses contemporains semblent tout droit émerger de cette distinction. Calliclès, en effet, dont Platon décrit le violent dialogue avec Socrate dans le Gorgias, présenta un discours moral en l'asseyant tout entier - faut-il croire - sur l'autorité d'une nature traversée par la domination des plus forts. Diogène, lui aussi coupable d'une critique acerbe à l'endroit de Platon et indirectement de son

1. Le terme «métaéthique » réfère aux questions de la nature de l'éthique, de ses limites, des méthodes qu'elle peut s'autoriser, etc. Même s'il n'est pas tel quel employé par les Grecs, le terme renvoie à des positions philosophiques qui, elles, sont bel et bien celles des Anciens.

2. Barney 2011.

3. Long et Sedley, vol. I, p. 29-33. Plus généralement, Brunschwig et Pellegrin montrent bien que l'épicurisme et le stoïcisme formaient des systèmes dont les différentes branches éthique et physique comprises - étaient reliées entre elles. 
maitré ${ }^{4}$, fit de même en calquant l'autarcie qu'il recherchait sur celle qu'il attribuait aux animaux. Comment expliquer que Diogène et Calliclès aient adopté des postures éthiques sinon antagonistes du moins divergentes, inspirées de ce qu'on pourrait, a priori, considérer comme la même nature? Il serait tentant de renvoyer dos à dos ces deux instances d'une même entreprise que l'on aurait tôt fait de juger naïve et trompeuse, voire funeste, entreprise qui entend tirer de la nature un discours moral. Mais, à la manière de Socrate - qui, même s'il ne croyait pas qu'un tel projet fût possible, s'attaqua, dans le Gorgias, non à la posture métaéthique de Calliclès, mais à sa façon de voir la nature ${ }^{5}$, il vaut la peine de s'attarder aux soubassements des positions de Calliclès et de Diogène. Après avoir montré à quel point leurs éthiques sont antagonistes, il faudra comparer leurs façons d'envisager la nature ainsi que les présupposés normatifs qui accompagnent, précèdent ou appellent leur observation de la nature.

\section{L'enseignement de la nature}

\section{A. Domination des autres, domination des circonstances}

\section{Calliclès : la leçon de la nature sur les meilleurs et les plus forts}

Lorsque Calliclès intervient dans la conversation entre Polos et Socrate pour demander si ce dernier plaisante lorsqu'il défend qu'il vaut mieux subir la pire injustice que la commettre (473b-d), il s'engage dans une lutte verbale qu'il sait d'avance violente. Sa thèse est en effet aux antipodes de celle de Socrate, dont les conséquences semblent à Polos contraires à l'opinion et à la nature (473c). Calliclès avance que « la justice consiste en ce que le meilleur ait plus que le moins bon et le plus fort plus que le moins fort » (483c, trad. Canto), à quoi il ajoute : « Partout il en est ainsi, c'est ce que la nature enseigne $(\delta \eta \lambda \sigma \tilde{)})$, chez toutes les espèces animales, chez toutes les races humaines et dans toutes les cités. » (483c.) Le fait que ce soit la nature qui nous «montre » quelque chose et non nous qui en « tirions » une leçon radicalise son propos. Ce n'est pas qu'une vision de la justice, mais celle de la nature. A priori, il semble que « pour Calliclès cette nature se ramène à l'appétit de domination : se conformer à la nature, c'est tendre à l' "emporter" » (483d $)^{6}$. La thèse de Calliclès peut être comprise de deux façons : d'abord, comme la conclusion valide des prémisses suivantes : l'observation de la nature dévoile la justice selon la nature; la nature doit être observée dans des domaines où les conventions n'ont aucune prise, c'est-à-

4. Cf. D.L. VI 25-26 [= SSR V B 55], 40 ; Élien, Histoire variée, IV 11 [= SSR V B 256].

5. Barney 2011.

6. Voelke 1982, p. 269. 
dire entre les États ou parmi les animaux ; l'observation de la nature montre que les plus forts dominent les plus faibles; donc, il est juste selon la nature que les plus forts dominent les plus faibles ${ }^{7}$. La deuxième façon ${ }^{8}$ élude la seconde prémisse, c'est-à-dire qu'elle considère que Calliclès inclut dans la nature les lieux où les conventions et les lois s'appliquent ${ }^{9}$. L'argument se trouve alors attaquable par ce qui donnera lieu à la suite de la conversation entre Socrate et Calliclès : si les meilleurs sont ceux qui dominent les autres, ne faudrait-il pas dire que la masse, en démocratie, est plus forte parce qu'elle impose ses lois à tous ${ }^{10}$ ?

Calliclès, pour qui la démocratie athénienne est un exemple patent d'injustice puisque les plus forts sont en réalité écrasés par les plus faibles et leurs lois, est forcé de se contredire :

Socrate: Alors, les lois établies par la masse ne sont-elles pas belles précisément parce qu'elles sont conformes à la nature - s'il est vrai que les êtres les plus forts sont les meilleurs?

Calliclès: Oui, j'affirme qu'elles sont belles conformément à la nature ! (488e.)

Il doit donc délaisser l'identité des forts et des meilleurs et préciser davantage ce qu'il entend par «meilleur », pour que le demos athénien n'en fasse pas partie (489e, 490a) : les meilleurs sont ceux qui possèdent la plus grande phronesis et c'est en vertu de cette différence avec la masse qu'ils peuvent la dominer et posséder plus qu'elle. Cette double thèse ne peut être admise qu'à moitié par Socrate : il peut accorder à Calliclès la supériorité de quelques aristoi ( $c f$. Rép. 484a), mais pas le fait que les meilleurs devraient posséder plus (Gorgias, 490b-c, Rép. 464b-c).

En butte aux tentatives incessantes de Socrate de le réfuter sur ce dernier point en montrant qu'un spécialiste ne devrait pas avoir un accès privilégié aux objets dont il est l'expert, Calliclès, comme le fera Diogène, accuse indirectement Socrate d'être mou :

D'abord, quand je parle d'êtres supérieurs, ils ne sont ni cordonniers ni bouchers! Non, je parle d'hommes intelligents, qui savent s'occuper des affaires de la cité [...] - des hommes qui non seulement sont intelligents, mais qui sont aussi courageux, assez forts pour accomplir ce qu'ils ont projeté de faire, et qui ne peuvent pas y renoncer par mollesse d'âme. (491a-b)

7. Barney 2011.

8. Stauffer 2002, p. 634. Calliclès aurait pu adhérer à la thèse d'Aristote, que la cité est un fait de nature et donc qu'elle en fait partie. Le fonctionnement interne des cités serait alors potentiellement pertinent pour en tirer son éthique.

9. La validité de l'argument de Calliclès repose donc sur sa définition de la nature, une question que nous aborderons plus tard ( $c f$. section II A.1).

10. Cette tentative de réfutation par Socrate pourrait être d'emblée bloquée, puisque le demos ne ferait dans ce cas pas partie de la nature. 
Le manque d'ambition politique, associé à la mollesse, est le défaut des intellectuels ${ }^{11}$, empêchés d'agir par leurs scrupules. Calliclès accuse les hommes raisonnables, qui « commande[nt] aux plaisirs et passions qui résident en [eux-mêmes] », d'être « des abrutis » (491e). Les scrupules d'un homme ne seraient que la manifestation de son incapacité de vivre en conformité avec la nature, à savoir dominer les autres et se laisser aller à ses passions ( $c f$. section I B. 1.) :

[Les hommes], qui sont eux-mêmes incapables de se procurer les plaisirs qui les combleraient, font la louange de la tempérance et de la justice à cause du manque de courage de leur âme. (492a.)

Ce faisant, Calliclès redéfinit une fois de plus les plus forts : ils sont courageux. C'est «la force de leur nature » qui leur permet de prendre le pouvoir (492b). Après une discussion enflammée sur l'hédonisme, Calliclès fait l'éloge de Périclès, Cimon et Thémistocle (503c) qui, ayant consacré leur vie au service de la cité, constituent le paradigme de l'homme courageux. Servir la cité serait donc une nouvelle caractéristique attribuable aux meilleurs (503a, c; 515c-d, 516b, 517a-b) ${ }^{12}$. Au final, Calliclès défend donc une conception de la justice naturelle selon laquelle les plus intelligents, courageux et forts sont les meilleurs, doivent posséder plus que les autres, les dominer et les diriger et donc faire carrière en politique.

\section{Diogène: vaincre les malheurs et les hommes}

L'éthique que Diogène tire de la nature est d'abord celle d'une forme d'autarcie particulière, c'est-à-dire l'adaptation aux circonstances :

Diogène vit un jour une souris qui courait çà et là, sans chercher de lieu de repos, sans prendre de précautions contre l'obscurité, et ne désirant rien de ce qu'on qualifie de jouissances : il y découvrit aussitôt [...] la façon de s'adapter aux circonstances. (D.L. VI $22=S S R$ V B 172)

Diogène est en effet exclu socialement et s'en lamente; il s'est déjà débarrassé de tous ses biens, mais il n'en a pas tiré de félicité (Plutarque, Moralia, 77E-78A). Devant le présumé bonheur de la souris, il comprend que le sien dépend de son attitude face à ce qui lui arrive. En fait, les conditions objectives de son bonheur sont naturellement déjà présentes et c'est la raison pour laquelle ce n'est pas d'abord sur elles que l'homme doit travailler. En effet, « la vie accordée aux hommes par les dieux est une vie facile, mais [...] cette facilité leur échappe » (D.L. VI $44=$ SSR V B 322). Les hommes doivent donc travailler sur leur façon de vivre, tirer parti de 
leur environnement, renouer avec cette facilité intime à l'animal ${ }^{13}$. C'est la voie courte vers le bonheur, ce « sentier de l'existence le plus facile à parcourir (D.L. VI $78=$ SSR V B 108) », que Diogène peut se targuer d'avoir emprunté.

Grâce au mode de vie qu'est la philosophie ${ }^{14}$ (D.L. VI $65=$ SSR V B 362) Diogène met en application l'enseignement de l'animal : lorsqu'on lui demande ce qu'elle lui a apporté, il répond «au moins ceci, sinon rien d'autre : je suis prêt à toute éventualité »(D.L. VI $63=$ SSR V B 360). Ainsi Diogène, comme les cyniques en général, mène une vie non téléo$\operatorname{logique}^{15}:$ devenir meilleur n'est point une destination, mais un processus, comme l'atteste la dynamique des épreuves (ponoi) qui caractérise la vie de Diogène ${ }^{16}$. Le ponos est ce que le philosophe de Sinope s'inflige constamment et activement afin de réactualiser sa capacité de s'adapter aux circonstances. À travers lui se confondent l'effort et le résultat de l'effort. Mais le ponos est aussi l'expérience initiale que Diogène vit peu avant de voir la souris ainsi que celle de son bannissement après qu'il eut falsifié la monnaie (D.L. VI $20=S S R$ V B 2), deux expériences qui l'arrachent au cadre habituel de son existence socialement intégrée. Ces ponoi permettent d'atteindre la connaissance de ce qui est naturel et du mode de vie véritable, car ce sont «tout aussi bien [des] mode[s] de connaissance que [des] mo$\operatorname{de}[\mathrm{s}] \mathrm{d}^{\prime} \operatorname{action}^{17} \gg$.

Cela dit, Diogène ne se contente pas de se dominer lui-même par les épreuves; il entend faire goûter sa médecine à ses concitoyens : «Ầ celui qui lui disait: "J'ai vaincu des hommes aux Jeux Pythiques", Diogène répondait : "Moi oui, j'ai vaincu des hommes; toi, des esclaves". » (D.L. VI $33=S S R$ V B 76.) Ce genre de comportement explique en partie son appellation de « chien »: il protégeait ses amis, les sauvegardait d'eux-mêmes (Stobée III 13, $42=$ SSR V B 307). L'éducation constituée par ce mode d'acquisition du savoir que constitue le ponos permet à Diogène d'aider les hommes à être naturels, ce qu'ils ne sont pas spontanément ${ }^{18}$. Il veut «les réveiller de leur léthargie bien pensante ${ }^{19} \gg$. Que ce soit pour lui ou pour les autres, Diogène, par les épreuves qu'il s'impose afin de demeurer en permanence insensible aux aléas du sort, adhère à la thèse de Socrate dans le Gorgias selon laquelle la souffrance rend l'homme meilleur (505b-c, 525b-

13. Husson 2011, p. 63.

14. Si conformément à Hadot cela est peut-être vrai de toute la philosophie grecque, dans le cas de Diogène la philosophie n'est peut-être qu'un mode de vie.

15. Kalouche 2003, p. 188.

16. Husson 2011, p. 64-67.

17. Husson 2011, p. 64.

18. Husson 2011, p. 54.

19. Goulet-Cazé 1992, p. 18. 
c), bien que sa mécanique et les circonstances de son application soient bien différentes. En ce sens, on peut parler d'une opposition radicale à Calliclès, pour qui toute injustice ou limitation est un esclavage (482b), non une école.

Cette façon de s'adapter aux circonstances diffère de la doctrine de Télès, selon laquelle chacun doit jouer le rôle que lui attribue la fortune ${ }^{20}$, puisque cette dernière philosophie prescrit de s'accommoder, sans broncher, de tout ce qui nous arrive, que ce soit d'être commandé ou de diriger les hommes, d'être comblé de richesses ou de connaître la pauvretée ${ }^{21}$. Diogène, au contraire, croit que certains rôles que pourrait « nous assigner »le destin doivent être délaissés. D’abord, le bonheur présent ne peut être parfait s'il est teinté de la crainte de le perdre dans le futur ${ }^{22}$ et il y a trop de risque de perdre la capacité d'adaptation aux circonstances si on accepte richesses, pouvoirs ou autres cadeaux empoisonnés. De plus, certaines situations où la liberté est réduite méritent qu'on y remédie : «Quelqu'un exaltait le bonheur de Callisthène d'avoir part à la magnificence d'Alexandre; "il est plutôt malheureux, reprit Diogène, lui qui déjeune et dîne quand il plaît à Alexandre" » (D.L. VI $45=S S R$ V B 30). Diogène préfère « falsifier la monnaie » (D.L. VI $20=S S R$ V B 2) que d'en accepter passivement les conséquences, il « oppose au destin la hardiesse » (D.L. VI $38=S S R$ V $B$ 7]). De ce fait, il se rapproche de l'idéal de Calliclès d'un homme qui aurait la nature suffisante pour bouleverser les conventions (484a).

\section{B. Hédonisme et ascèse}

\section{Calliclès : le tonneau percé}

La domination des meilleurs ne saurait être bien comprise sans en préciser le motif, soit l'assouvissement perpétuel des passions en conformité avec la nature :

Calliclès : Veux-tu savoir ce que sont le beau et le juste selon la nature ? Hé bien, je vais te le dire franchement! Voici, si l'on veut vivre comme il faut, on doit laisser aller ses propres passions, si grandes soient-elles, et ne pas les réprimer. Au contraire, il faut être capable de mettre son courage et son intelligence au service de si grandes passions et de les assouvir avec tout ce qu'elles peuvent désirer. (492a.)

Pour Calliclès, la nature nous apprend à asservir notre intelligence et notre courage au profit de nos passions. Conséquemment, cultiver leurs

20. Télès, fr. II, p. 5, 2-6, 8 Hense ( $c f$. Fuentes González 1998, p. 34-35, 148 sqq.). Voir aussi $S S R$ V B $46 ; 469 ; 222 ; 245$.

21. Télès, fr. VI, p. 52, 2-5 Hense (cf. Fuentes González 1998, p. 468-469, 475). Voir aussi $S S R$ I C 512-513.

22. Goulet-Cazé 1992, p. 63. 
désirs pour pouvoir les combler par la suite constitue la visée de la domination des plus forts. C'est l'action de combler leurs besoins qu'ils doivent rechercher, non la satiété. Les individus sans besoins ne peuvent donc pas être heureux : s'il était vrai que les hommes sans besoins étaient heureux, «les pierres et même les cadavres seraient tout à fait heureux » (492e). Ce contre-exemple met en relief non seulement l'impossibilité du bonheur pour de tels hommes, mais le fait que ce ne sont pas vraiment des hommes $^{23}$. Que reste-t-il à l'homme une fois ses plaisirs retirés, sinon la vie intellectuelle ? Assimiler le penseur à un cadavre serait alors une marque d'anti-intellectualisme. Il serait pourtant possible de considérer que même la vie intellectuelle consiste en la poursuite de besoins et l'effort pour les combler. Le philosophe aurait de grands besoins, ce qui n'est pas étranger à l'aspiration du philosophe évoquée par Diotime (Banquet, 210e-212a).

La différence entre l'idéal d'autarcie cynique et le rejet de l'autarcie par Calliclès est criante : d'un côté, Diogène rappelle que « s'il appartient aux dieux de n'avoir besoin de rien, il appartient aux gens semblables aux dieux d'avoir des besoins limités »(D. L. VI $104=S S R$ V A 135); de l'autre, Calliclès ne peut admettre que les dieux sont sans besoin, sans quoi il les aurait comparés à des pierres. Cela dit, la dynamique que Calliclès propose est tout de même semblable à celle des ponoi, c'est-à-dire qu'il y a provocation, volontaire ou non, d'une souffrance pour engendrer une joie concomitante. Chez le cynique, l'épreuve engendre la joie apportée par le sentiment de libération à l'égard des plaisirs ${ }^{24}$; chez Calliclès, ces souffrances ne sont autres que les désirs en attente d'être comblés : « la faim est une chose pénible. Malgré tout, manger quand on a faim, c'est bien agréable! » (496c.) C'est pourquoi Socrate compare le mode de vie de plaisirs proposé par Calliclès à un tonneau percé, que l'homme menant une telle vie serait sans cesse occupé à remplir, « en s'infligeant les plus pénibles peines » (493e, 494a) et que Calliclès rétorque que « la vie de plaisirs est celle où l'on verse et reverse autant qu'on peut dans le tonneau » (494a), un tonneau qui comporte « de gros trous » (494a).

Devant l'échec de Socrate à convaincre son interlocuteur par des métaphores (493d), il ridiculise sa position en montrant qu'elle ne peut que prescrire les vies du pluvier et du kinaidos ${ }^{25}$ (494e), et entame une réfutation sur la différence entre le bien et le plaisir (495a-499b). Calliclès est contraint d'accepter qu'il y a une hiérarchie entre les plaisirs (499b), mais il se refuse à admettre explicitement que les plaisirs sont subordonnés au bien. Cependant, ce point ne mine pas la thèse de Calliclès :

23. Canto 2007, p. 339, n. 132.

24. Husson 2011, p. 71. Pour une comparaison détaillée sur la question du plaisir chez Diogène et Antisthène, $c f$. Brancacci 1993.

25. Monoson 2000, p. 164. 
[His] philosophical enthusiasm is not, it seems, for pleasure itself but for the intensity, self-assertion and extravagance that accompany its pursuit on a grand scale: he endorses hedonism so as to repudiate the restraints of temperance, rather than the other way around ${ }^{26}$.

De fait, même s'il acceptera de hiérarchiser les plaisirs, jamais Calliclès ne renoncera à soutenir que la tempérance (sophrosyne) et la justice conventionnelle sont «mauvaises et vilaines selon la nature » pour ceux qui pourraient s'emparer du pouvoir (492c). Si l'homme qui subit l'injustice sans pouvoir se porter assistance à lui-même est un esclave (483b) et qu'empêcher le meilleur d'entretenir et de combler ses désirs est une injustice selon la nature, alors la masse, lorsqu'elle dévalorise l'entretien des désirs, « réduit à l'état d'esclaves les hommes dotés d'une plus forte nature $\gg(492 \mathrm{a})$.

\section{Diogène : le tonneau habité}

Diogène défend lui aussi une philosophie de la liberté. Cela dit, si Calliclès veut se débarrasser de l'asservissement à autrui en le dominant et en laissant libre cours à ses passions, Diogène entend gagner sa liberté bien autrement. Au «dérèglement » et à « la liberté de faire ce qu'il veut » (492c), Diogène oppose une liberté fondée sur une véritable ascèse (askesis), sur l'exercice :

Rien, disait-il, n'a de chance de réussir dans la vie sans entraînement, et c'est l'entraînement qui est capable de tout surmonter. Il faut donc préférer à des efforts inutiles ceux que recommande la nature pour en arriver à vivre heureux: c'est par leur bêtise même que les hommes se rendent malheureux. En effet, le mépris du plaisir lui-même est très agréable quand on s'y est entraîné $[. .$.$] . Voilà quels étaient ses discours et il les démontrait en$ actes $[. .$.$] n'accordant jamais à la coutume le poids qu'il donnait aux valeurs$ naturelles : en ses propres mots, il menait le genre de vie qui avait caractérisé Héraclès quand il mettait la liberté au-dessus de tout. (D. L. VI 71 $=S S R$ V B 291.)

Par l'askesis, Diogène parvient à résister à la douleur en s'imposant des ponoi comme se rouler dans le sable brûlant et marcher pieds nus dans la neige (D. L. VI $23 ; 34=S S R$ V B 174; 176) : il atteint ainsi la résistance, la karteria. Il apprend à mépriser les plaisirs et à limiter ses besoins, ne désire que l'essentiel et se maîtrise lui-même (enkrateia) de ce fait (D. L. VI 55; $104=$ SSR V B 441). À cette fin, il élimine vêtements luxueux - il double son manteau (D. L. VI $22=S S R$ V B 174) -, nourriture élaborée ou rare et ustensiles de cuisine (D. L. VI 37, $44=S S R$ V B 158, 322), mais aussi soif de gloire et maison (D. L. VI $104=S S R$ V A 135). Diogène ex- 
tirpe donc peu à peu ses besoins et atteint une relative autarcie (autarkeia). Ce passage, qui montre bien le lien étroit entre enkrateia et autarkeia, est saisissant: le processus auquel s'adonne Diogène est en fait une réelle guerre intérieure ${ }^{27}$ pour gagner sa liberté.

S'il s'impose quelque chose à lui-même, comment peut-il être libre ? ne manquerait pas de lui demander Calliclès. Diogène répondrait que l'homme est un, corps et esprit ${ }^{28}$, et que la raison n'est pas plus une force étrangère que les passions; que ce sont en fait les passions qui sont imposées de l'extérieur, engendrées qu'elles sont pour la plupart par la vie en société (D. L. VI $44=S S R$ V B 322). Au contraire, par nature, l'homme est autarcique. C'est dire que, pour Calliclès, les passions sont naturelles et leur limitation conventionnelle; pour Diogène, la limitation des besoins est naturelle et la majorité des passions conventionnelle! Héraclès, modèle pour Calliclès comme pour Diogène, est admirable, pour le premier, à cause de la force avec laquelle il domine Géryon et ses bœufs (484b), mais, pour le second, à cause de sa résistance et de son enkrateia et du courage dont il a besoin pour l'exercer. C'est ainsi qu'à « celui qui tirait vanité de la peau de lion qui le couvrait [le vêtement que portait Héraclès ([Lucien], Le Cynique, 13)], Diogène disait : "Cesse donc de déshonorer le vêtement du courage” » (D. L. VI $45=S S R$ V B 465). Pour Diogène, l'idéal hédoniste de Calliclès est plutôt le paroxysme de la mollesse, mollesse que Calliclès dénonce lui aussi ! C'est que, pour l'interlocuteur de Socrate, la mollesse caractérise l'intellectuel qui abandonne ses ambitions (491b), alors que chez les cyniques elle est le propre de celui qui est sans « constance ni empire sur [lui]-même $\gg$ (Cratès, Lettre $34=$ SSR V H 121).

Par ailleurs, même s'il lui est laborieux d'atteindre l'autarkeia, une autre facette de l'aptitude à s'adapter aux circonstances, l'homme n'a pas besoin des autres, si ce n'est de quelqu'un qui lui inflige un ponos pour le réveiller de sa vie de mollesse $e^{29}$. La lumière se fait alors sur un passage que nous avons analysé plus haut (cf. Section I, A. 2.) :

Il répétait à cor et à cri que la vie accordée aux hommes par les dieux est une vie facile, mais que cette facilité leur échappe, car ils recherchent gâteaux de miel, parfums et raffinements du même genre. (D. L. VI $44=S S R$ V B 322.)

La vie est facile puisque les faux besoins de l'homme n'ont pas à être comblés pour qu'il atteigne le bonheur. L'autarkeia, et avec elle l'enkrateia et la karteria, sont les véritables ingrédients de la félicité. Encore une fois, Calliclès et Diogène utilisent une même notion positivement - «vivre

27. Husson 2011, p. 55.

28. Goulet-Cazé 1986, p. 12.

29. Cf. Fuentes González 2003. 
dans la facilité » permet pour tous les deux la vertu et le bonheur (492c) (D. L. VI $44=$ SSR V B 322) -, mais lui attribuent un sens différent. La facilité de Calliclès renvoie à l'absence de tempérance et de difficulté à combler des besoins débordants; Diogène l'emploie pour référer à la possibilité d'une vie simple et frugale. Voilà qui complète notre analyse: Diogène et Calliclès proposent des éthiques opposées reposant chacune sur le recours à la nature ${ }^{30}$.

II. La délimitation de la nature et la perspective adoptée sur elle

\section{A. L'étendue de la nature}

1. Calliclès : englober les cités dans la nature, encenser certains hommes et observer leurs rapports

Il n'est pas aisé de déterminer quelles sont les limites exactes de la nature pour Calliclès. Nous avons vu précédemment que les exemples qu'il donne en $483 \mathrm{~d}$ et $484 \mathrm{~b}$ relèvent de lieux où les conventions humaines sont absentes : le terrain de la politique internationale (l'exemple de Darius et de Xerxès) et celui des territoires lointains (celui de Géryon). Les conventions ne feraient alors pas partie de la nature, ce qui est d'autant plus plausible que Calliclès les dénigre systématiquement et les oppose à la nature. Et pourtant, lorsqu'il dit: « partout il en est ainsi, c'est ce que la nature enseigne, chez toutes les espèces animales, chez toutes les races humaines et dans toutes les cités » (483c), il inclut dans la nature non seulement le règne animal, mais aussi celui des hommes. C'est qu'en fait les hommes font bel et bien partie de la nature, même ceux qui vivent en cité, mais les conventions elles-mêmes ne sauraient être associées à des faits naturels. Cela concorde avec les propos de Calliclès d'une part lorsqu'il affirme que l'homme est un animal parmi d'autres (516b) et d'autre part lorsqu'il encense des hommes d'État athéniens comme Périclès (503c) - hommes qui par ailleurs s'illustrèrent tous par l'éclat de leurs actions en politique internationale ${ }^{31}-$ ce à quoi s'est toujours refusé Diogène ${ }^{32}$. On comprend aussi l'exhortation de Calliclès à «imit[er] les citoyens qui ont une vie de qualité, une excellente réputation et jouissent de tous les autres bienfaits de l'existence » (486d).

Si Calliclès accepte d'intégrer les hommes parmi la nature, il ne fait pourtant pas de chacun d'eux un modèle que sa doctrine naturaliste in-

30. Le recours à la nature par Calliclès et Diogène n'est donc pas d'abord rhétorique, comme nous en avons fait une démonstration parallèle dans Chevarie-Cossette 2013.

31. Canto 2007, p. 346-347, n. 171-174.

32. Diogène admirait au contraire ceux qui s'apprêtaient à se lancer en politique, mais changeaient d'avis (D. L. VI $29=S S R$ V B 297). 
citerait à imiter. L'interlocuteur de Socrate refuse, tout comme Hippias, Critias et Alcibiade ${ }^{33}$, d'être comparé à des hommes « inférieurs » comme des médecins, des bouchers ou des cordonniers (490c; 491a). Cela s'explique simplement parce que ces professions n'incarnent pas les idéaux de force, de phronesis et de courage. De fait, le rejet par Calliclès du pluvier (494b), du kinaidos (494d), mais aussi du sophiste (520a) ne peut être expliqué par le lien entre ces exemples et la cité, puisque le pluvier est un animal, le kinaidos n'est pas accepté socialement et le sophiste est communément accusé de corruption de la jeunesse ${ }^{34}$. C'est plutôt respectivement en vertu du manque de force, de la position de dominé adoptée et de l'intellectualisme ${ }^{35}$ que le pluvier, le kinaidos et le sophiste sont écartés comme modèles éthiques. Être hors norme est donc nettement insuffisant pour devenir un modèle à imiter; au contraire, l'idéal de la nature, c'est plutôt «le tyran impitoyable $\gg^{36}$.

En plus de ce filtre évident, que Calliclès utilise pour sélectionner ce qui, dans la nature, concorde avec sa théorie - une manœuvre qu'on aura tôt fait de détecter chez Diogène -, Calliclès adopte un point de vue particulier sur les exemples qu'il a sélectionnés. Il n'utilise ni les propriétés de ses modèles ni le rapport de ceux-ci à eux-mêmes - comme ce sera le cas chez Diogène -, mais le rapport de domination entre le modèle et la masse et de possession entre celui-là et les objets. Si Diogène avait eu à observer Xerxès et Darius, il aurait d'abord remarqué leur absence d'autarcie et leur orgueil ; Calliclès s'intéresse à leurs actions et donc à leur rapport avec les autres: « Eh bien, [Xerxès] et son père ont agi, j’en suis sûr, conformément à la nature du droit ${ }^{37}$ - c'est-à-dire conformément à la loi, oui, par Zeus, à la loi

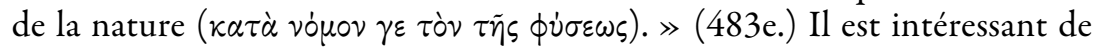
noter que, pour Calliclès, ce que la nature enseigne, ce n'est pas «comment être le meilleur », mais «ce que doit posséder (matériellement) le meilleur » (483d). Lorsque Calliclès affirme que nous devons vivre nos passions (492a) - si R. Barney a raison d'affirmer que « [he] leave[s] the content of those appetites entirely a matter of subjective preference ${ }^{38} \gg$ et que le rejet de la tempérance est plus un rejet de toute forme de contrôle qu'une exhortation aux plaisirs ( $c f$. section I B. 1) - l'enseignement de la nature concerne encore une fois le rapport de domination entre soi et les autres.

33. Canto 2007, p. 337, n. 120.

34. Canto 2007, p. 356, n. 237.

35. Barney 2011.

36. Canto 2007, p. 73.

37. La nature du droit est en même temps un droit selon la nature chez Calliclès: « Agir "selon la nature du droit", c'est agir "selon la loi de la nature", et cette loi - qui n'a rien d'une convention - est appelée aussi le "droit de la nature" » (Voelke 1982, p. 268).

38. Barney 2011. 
En l'absence de l'adhésion de Calliclès à une position allant dans le sens de la division de l'âme et de l'identité de l'homme avec son logos (cf. Rép. $588 \mathrm{~d}$ ), il est plausible de croire que pour Calliclès, la tempérance n'est pas tant une maîtrise de soi que l'imposition de quelque chose d'étranger (les conventions qui provoquent les scrupules chez l'intellectuel [491b]) sur ce qui est premier chez l'homme (ses passions).

\section{Diogène : délimiter la nature}

en fonction de la relation de l'homme à sa nature

Diogène, comme Calliclès, délimite la nature à sa façon. Celle-ci semble a priori ne pas inclure les hommes ou, du moins, pourrait-on croire initialement, ce dont s'inspire Diogène pour ériger son éthique, ce sont les animaux et eux seuls, animaux qui vivent la facilité offerte par les dieux et qui sont en pleine possession de leur nature ${ }^{39}$. Néanmoins, force est de constater que la nature ne se limite pas aux animaux. Un premier indice de ceci est l'anecdote suivante: «voyant un jour un petit garçon boire dans ses mains, il [Diogène] jeta son gobelet hors de sa besace en s'écriant : "un gamin m’a dépassé en frugalité !” » (D. L. VI 37 = SSR V B 158.) Qu'ont en commun l'animal et l'enfant ? Ils ne sont pas socialisés, moulés par les conventions. C'est cette propriété qui sert à discriminer ce qui est naturel ou non. Par conséquent, un homme qui se détournerait des conventions habiterait la nature; la souris de ville aussi, alors que Diogène, avant sa conversion, n'était pas encore un homme ${ }^{40}$. Pour l'homme, réintégrer la nature signifie qu'il réalise la sienne; c'est que «l'homme naturel » signifie « l'homme réalisé ${ }^{41}$. Cette réintégration doit être renouvelée, par les ponoi : «l'homme, en tant que partie de la nature, possède une nature, mais qu'il doit perpétuellement gagner et reconquérir, comme s'il devait démontrer qu'il [...] est lui-même à la hauteur de son humanitét ${ }^{42}$. » Justement, cette quête de l'humanité est primordiale pour Diogène : il la cherche en plein jour avec une lanterne (D. L. VI $41=S S R$ V B 272), parmi une foule où il ne voit que des déchets (D. L. VI $32=S S R$ V B 278), aux Jeux olympiques et au bain public où la foule était grande, mais les hommes peu nombreux (D. L. VI 40; 60 = SSR V B 274;273), dans toute la Grèce où il ne trouve aucun homme de bien (D. L. VI $27=S S R$ V B 280).

Quelle est la perspective de Diogène sur cette nature qui inclut les animaux et les hommes qui se sont extirpés des conventions ? À rebours de Calliclès qui observe dans la nature les rapports entre ses membres, Diogène porte son regard sur le rapport des individus naturels (animaux ou en-

39. Husson 2011, p. 11.

40. Husson 2011, p. 71.

41. Adams 1945, p. 97.

42. Adams 1945, p. 10. 
fants) à leur environnement et aux circonstances que leur présente leur existence. Diogène s'intéresse en effet à la question de la manière dont il faut vivre sa vie bien avant celle de la justice entre les hommes. C'est pourquoi ses modèles sont toujours un animal solitaire ou un enfant seul (D. L. VI $22 ; 37=S S R$ V B $172 ; 158)$, des exemples qui n'ont rien à voir avec les Xerxès, Darius et Périclès de Calliclès.

\section{B. Modèle animal, modèle divin}

\section{Calliclès : la fable du lion et les boufs de Géryon}

Nous avons vu précédemment que Calliclès rejetait le modèle animal du pluvier (494b). Les seules autres mentions d'un animal dans son dialogue avec Socrate sont le lion, dans une métaphore, et un peu plus loin les bœufs, dans le mythe d'Héraclès. Il n'y a donc aucun exemple, comme chez Diogène, d'animal que Calliclès observe directement dans la nature et duquel il déduit des prescriptions. L'allusion au lion demeure malgré tout très informative sur la position de Calliclès :

Chez nous, les êtres les meilleurs et les plus forts, nous commençons à les façonner, dès leur plus jeune âge, comme on fait pour dompter les lions ; avec nos formules magiques et nos tours de passe-passe, nous en faisons des esclaves, en leur répétant qu'il faut être égal aux autres et que l'égalité est ce qui est beau et juste. Mais j'en suis sûr, s'il arrivait qu'un homme eût la nature qu'il faut pour secouer tout ce fatras, le réduire en miettes et s'en délivrer, si cet homme pouvait fouler aux pieds nos grimoires, nos tours de magie, nos enchantements, et aussi toutes nos lois qui sont contraires à la nature - si cet homme, qui était un esclave, se redressait et nous apparaissait comme un maître, alors, à ce moment-là, le droit de la nature brillerait de tout son éclat. (483d-484a.)

Calliclès compare la jeunesse athénienne à des lions asservis, c'est-à-dire incapables de se défendre d'une injustice selon la nature (483b), et donc de cultiver librement leurs désirs. Ce qui est enseigné aux enfants leur est ici répété et non prouvé; un vocabulaire péjoratif associe l'éducation, le savoir et les conventions à des artifices («formules magiques », « tours de passepasse », « tours de magie », « enchantements »).

La suggestion de M. Canto, à l'effet que cette métaphore ferait référence à la fable d'Eschyle reprise par Aristophane ${ }^{43}$ semble des plus pertinentes, surtout que celle-ci ressemble aux Grenouilles, pièce où Aristophane dénonce la soumission d'Athènes aux caprices d'Alcibiade ${ }^{44}$. En effet, dans la fable d'Eschyle, le chœur raconte la parabole du lion qui, adopté pour accroître les richesses d'une maison, en engloutit le troupeau.

43. Canto 2007, p. 332, n. 89.

44. Judet de la Combe 1982, p. 62. 
Malgré les tentatives de la famille de dompter le fauve, elle échoue à changer sa nature, qui finit par ressurgir. Le parallèle est probant : Calliclès dénonce le travestissement des jeunes Athéniens - tout comme la fable dénonce celui du lion - et formule un présage : un individu disposant de

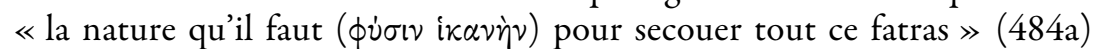
renverserait les conventions comme le lion dévorant le troupeau ${ }^{45}$. La fable rappelle le caractère artificiel des conventions, c'est-à-dire qu'elles ne font que donner l'impression aux faibles qu'ils sont les égaux des plus forts ( $c f$. 483c). C'est réellement une fable anticonventionnaliste et naturaliste que présente Calliclès, puisqu'elle réaffirme l'interdiction de transgresser la nature tout en réitérant la supériorité et l'inexorabilité de celle-ci. Mais cette loi naturelle ne saurait être réduite à une triste fatalité : le résultat du renversement des normes par un homme au naturel fort - « le droit de la nature brillerait de tout son éclat $\gg(484 a)$ - est le résultat d'une libération («si cet homme se redressait », 484a).

La deuxième occurrence d'un animal dans l'entretien du Gorgias est en elle-même banale, mais peut servir à mieux distinguer la position de Calliclès de celle de Diogène, surtout lorsqu'elle est lue dans son contexte, puisqu'elle précise en quoi consiste la présence des dieux dans la nature :

Pindare $[\ldots]$ exprime les mêmes idées que moi dans $[$ son $]$ ode $[\ldots]:$ « la loi, reine du monde, des êtres mortels et des dieux immortels, $[\ldots]$ conduit le monde d'une main souveraine, pour justifier la plus extrême violence ; j'en veux pour preuve les travaux d'Héraclès : sans rien payer... » [...]; en tout cas, il dit qu'Héraclès a pris les bœufs de Géryon, sans avoir payé Géryon, sans que celui-ci, non plus, lui en ait fait cadeau, certain que c'est bien là le droit de la nature, que les bœufs et tous les autres biens des êtres inférieurs et plus faibles appartiennent en entier à l'homme qui leur est supérieur en force et en qualité. ( $484 \mathrm{~b}-\mathrm{c}$.)

Nous avons la confirmation que lorsque Calliclès mentionne la nature, il présente les rapports de domination entre ses membres, dans le cas présent entre Héraclès et Géryon (Héraclès domine Géryon pour le voler), entre Héraclès et les bœufs (Héraclès les asservit) et, de manière générale, entre les êtres inférieurs et les hommes (ceux-là sont possédés par ceux-ci). Tous ces rapports sont conformes à la loi de la nature, loi qui s'impose également aux « dieux immortels ». Or, si les dieux sont réellement soumis à la loi du plus fort, il n'est pas étonnant que Calliclès ne pense pas aux dieux lorsqu'il compare les êtres sans besoins à des pierres (482e). En effet, les dieux ont ici toutes les chances de correspondre à la description qu'en fait Hésiode, le poète notamment à l'origine du mythe des douze travaux d'Héraclès, auquel Calliclès fait référence. Puissants et dominateurs, ils

45. Id. p. 61. 
sont agités par leurs passions. Cette divergence de la conception du divin est peut-être en partie à l'origine de divergences éthiques entre Calliclès et Diogène, pour qui les dieux sont sans besoins (D.L. VI $104=S S R$ V A 135). Le choix d'Héraclès et de ses actions violentes ${ }^{46}$ est lui aussi porteur de signification : contrairement à la tradition cynique, pour qui Héraclès est un modèle de maîtrise de soi, le demi-dieu est ici avant tout modèle de force et d'ambition.

$\mathrm{Au}$ demeurant, il est assez clair ici que la tripartition classique dieuxhumains-animaux et la hiérarchie qui s'y applique traditionnellement demeure intacte, les animaux n'étant pour Calliclès que des objets à posséder.

\section{Diogène : petits animaux, grands dieux}

Il reste deux questions essentielles à élucider concernant la nature circonscrite par Diogène pour déduire une éthique : d'abord, la question de la sélection des animaux; ensuite, celle de la caractérisation des dieux. Diogène doit en effet sélectionner certains animaux; sa démarche ne consiste pas « à imiter aveuglément l'animal, quel qu'il soit, mais à réaliser en soi-même et à développer dans toute sa dimension et toutes ses conséquences la vraie nature de l'homme : l'exemple animal n'est pas le but du chemin qui conduit à la vertu et au bonheur; il en est un point de passage $^{47} \gg$. Diogène observe successivement une souris (D. L. VI $22=S S R$ V B 172), un escargot (Lettre $16=S S R$ B 546), et des souris (D. L. VI 40$=S S R$ V B 173). D'autres bêtes sont mentionnées, mais elles ne sont pas à proprement parler «observées »: la peau d'un lion pour symboliser le courage (D. L. VI 45; $75=S S R$ V B 465; IV B 70 $0^{48}$ ), la tarentule et le scarabée (ou escarbot) pour caractériser le refus violent de Perdiccas de recevoir Diogène, le nom de « chien » qu'il emploie (D. L. VI 44;33 = SSR V B $50 ; 144)$, le poulpe cru qu'il aurait peut-être mangé et dont il serait mort (D. L. VI $76=S S R$ V B 94), un hareng dont il se sert pour briser les conventions (D. L. VI $36=$ SSR V B 367). Tous les animaux retenus sont petits, solitaires et sans grands besoins. En outre, certains animaux seraient rejetés en vertu de leur relation avec la nature qui les entoure :

Pour Cratès comme pour Diogène, l'escarbot n'est [...] pas un bon exemple de conduite pour l'homme. La fourmi ne saurait l'être davantage. Dans la fable d'Ésope, la cupidité de la fourmi [...] représente tout ce qui s'oppose à l'autarcie. La fourmi symbolise l'homme qui, parce qu'il est incapable de se

46. Calliclès admire la rhétorique et sa violence, dont il fait usage durant le dialogue (485c-d ; 486c).

47. Flores-Junior 2005, p. 163.

48. $C f$. aussi Maxime de Tyr, XXXVI 5-6 = SSR V B 299, et SSR IV B 98. 
contenter de ce qui lui est propre, regarde d'un œil d'envie le produit du travail des autres ${ }^{49}$.

La souris et l'escargot, par ailleurs, dans leur rapport à leur environnement, sont des modèles : alors que la souris comprend comment s'adapter aux circonstances, l'escargot n'a pas besoin d'autre abri que luimême. L'ambivalence de l'utilisation des animaux reste un signe que le cynique « adoptai $[t][. .$.$] une véritable "vie de bête", dictée pourtant par l'in-$ telligence humaine $e^{50} \gg$ puisque l'appropriation de la nature par Diogène se fait toujours à travers le filtre de la raison.

En ce qui concerne les dieux, nos sources sont on ne peut plus claires sur la conception que s'en faisait Diogène : «s'il appartient aux dieux de n'avoir besoin de rien, il appartient aux gens semblables aux dieux d'avoir des besoins limités » (D. L. VI $104=S S R$ V A 135). Cela est important puisque le cynique tend vers l'autarcie divine: « [de l'avis de Diogène], les hommes de bien sont les images des dieux $\gg$ (D. L. VI $51=$ SSR V B 354). Cette quête d'assimilation à la divinité, qui emprunte parfois la voie de l'imitation d'Héraclès, comme on l'a vu (D. L. VI $71=S S R$ V B 291), semble ne pas avoir suffi au cynique. Diogène, en effet, se tourne vers les animaux et en fait d'autres modèles, en plus des dieux, ce qui vient, d'une certaine façon, remettre en cause la tripartition classique entre les hommes, les dieux et les animaux. C'est peut-être qu'il a conscience que prescrire l'autarcie sur la base de celle des dieux est une fondation doublement fragile. D'abord, si l'on croit que les dieux sont autarciques, c'est bien parce qu'on y voit une qualité. L'appel aux animaux évite donc (peut-être) une forme de circularité. Ensuite, l'idéal d'une intimité avec la facilité propre à la vie naturelle que Diogène attribue aux animaux est plus accessible et con$\operatorname{cret}^{51}$ que celui de dieux inaccessibles, invisibles ${ }^{52}$.

Quant à la différence de valeur entre l'animal et l'homme, elle est plus laborieuse à établir. L'animal, bien sûr, sert de modèle - il a réalisé sa propre nature contrairement à la plupart des hommes -, mais cette différence est probablement contingente : les hommes pourraient bel et bien être autarciques. Il faudrait alors croire que « selon le cynique, l'animal n'est pas supérieur à l'homme », mais que « [la plupart] des hommes, parce qu'ils ne savent pas mettre à profit toutes leurs capacités, sont inférieurs aux ani$\operatorname{maux}^{53} \gg$. Quoi qu'il en soit, il est nécessaire de mettre en relief le fossé qui sépare sur cette question Calliclès et Diogène. Celui-là semble réduire les

49. Flores-Junior 2005, p. 154

50. Id. p. 161.

51. Goulet-Cazé 2005, p. 10-11.

52. C'est cette idée que défend Protagoras: ne voyant pas les dieux, nous ne pouvons nous en inspirer.

53. Flores-Junior 2005, p. 163. 
animaux, aux rares moments où il s'y intéresse, à des objets potentiels de domination et de possession (484c) alors que Diogène les érige en modèles. Il est compréhensible, pour une raison de plus, que leurs éthiques, quoique toutes les deux basées sur la nature, divergent fondamentalement. Seule similitude, la figure du lion - qui rappelle celle d'Héraclès - représente l'attachement des deux hommes au courage (Plat. Gorg. 484 b et D. L. VI $45=S S R$ V B 465).

III. Les positions qui accompagnent, précèdent ou incitent à l'observation de la nature

\section{A. De l'anticonventionnalisme au naturalisme}

\section{Calliclès : généalogie de la morale et amour de la liberté}

Malgré son apparence d'immoralisme ${ }^{54}$, Calliclès croit bel et bien à une justice supérieure ${ }^{55}$, laquelle se trouve non dans les lois, mais dans la nature $^{56}$. L'interlocuteur de Socrate est le défenseur d'une morale anticonventionnaliste ${ }^{57}$. Il faut croire également que la démarche même de la déduction d'une éthique à partir de la nature procède, elle aussi, de son anticonventionnalisme, qu'il faut déceler dans sa critique de l'origine des lois et dans l'importance qu'il accorde à la liberté, surtout celle de la parole.

Calliclès critique l'origine des lois et des conventions, élabore une généalogie de la morale pour fonder son éthique, une méthode propre à plusieurs sophistes ${ }^{58}$. Dès lors que Calliclès a fait la preuve que la loi et la nature se contredisent le plus souvent $(482 \mathrm{e} ; 492 \mathrm{c})$ et que la nature est plus vraie que la loi, la table est dressée pour qu'il puisse exposer son éthique. La priorité de la nature sur la loi est déjà contaminée par son anticonventionnalisme : les manières, les conventions sont faites par les hommes à l'encontre de la nature; ce ne sont que « des paroles en l'air, qui ne valent rien » (492c). Comme d'une part la loi est toujours à l'image des motivations de ceux qui la créent et que ceux-ci sont en réalité les plus faibles, dont le souhait est d'《 avoir l'air d'être égaux » (483c), la loi n'est qu'une apparence. En réalité, certains hommes sont supérieurs aux autres (483d) malgré l'isonomia. L'utilisation de la source des conventions pour les discréditer est présente dans deux autres passages : 
C'est pour empêcher que ces hommes [scil. les plus forts] ne leur soient supérieurs qu'ils [scil. la masse] disent qu'il est vilain, qu'il est injuste, d'avoir plus que les autres et que l'injustice consiste justement à vouloir avoir plus. (483c.)

La masse blâme les hommes qui vivent ainsi, gênée qu'elle est de devoir dissimuler sa propre incapacité de le faire [...] Ces derniers [scil. la masse], qui sont eux-mêmes incapables de se procurer les plaisirs qui les combleraient, font la louange de la tempérance et de la justice à cause du manque de courage de leur âme. (492a.)

Ces deux «arguments de l'origine», qui consistent à considérer qu'une action est illégitime parce qu'elle est motivée par la crainte, la jalousie ou la faiblesse, sont au cour du naturalisme de Calliclès. Si l'observation de la nature nous révèle ce que sont réellement les choses, alors la description naturelle d'une chose en épuise la valeur. Autrement dit, Calliclès croit que toutes les conventions peuvent être réduites à leur description «naturelle ». Dès lors que la motivation naturelle à l'origine d'une loi est méprisable, cette loi l'est tout autant. Et, comme Calliclès a une vision extrêmement critique des ces motivations - ce qui procède de son anticonventionnalisme -, il ne peut que rejeter les lois et les conventions qui en découlent. On le voit clairement dans le deuxième exemple, où il attribue la naissance des lois à l'incapacité de mener une vie hédoniste. Bref, sa position éthique, issue de la priorité qu'il accorde à la nature sur les lois, tire son origine plus profondément de son anticonventionnalisme au sens de son mépris de lorigine des conventions. Par opposition, la nature semble pour Calliclès - dès lors que les conventions sont discréditées - caractériser ce que sont les choses plus profondément : par exemple, Calliclès passe de «subir une injustice est laid selon la nature, non selon la loi » à « l'homme qui subit l'injustice n'est pas un homme » (483a-b). Autrement dit, quand Calliclès parle de l'essence de l'homme, il suppose d'emblée qu'il s'agit de son essence selon la nature $e^{59}$. Se réaliser comme homme est d'abord une question de nature, non de conventions.

Ce que comprend Calliclès sous le mot de liberté est aussi l'une des causes probables de son entreprise naturaliste. La liberté se comprend pour lui comme négative : c'est « la liberté de faire ce qu'on veut » (492c), c'està-dire l'absence de contrôle des désirs d'un individu par les conventions. Supporter « les lois, les formules et les blâmes de la masse des hommes » serait «se mettr[e] [soi]-même un maître sur le dos » (492b). D'ailleurs, les mécanismes de contrôle des désirs sont extrêmement dépréciés par Calliclès (483b, 483d, 485c, 492a). C'est dire que les désirs sont naturels pour Calliclès; ils ne viennent ni de la cité ni de la mollesse. La nature, en effet, englobe la totalité du monde à l'exception des conventions. Or, celles-ci ne 
peuvent provoquer des changements dans les désirs, car elles ne changent que les apparences, conformément à l'enseignement de la fable du lion (484a). Donc, si les désirs sont entièrement naturels et que la liberté, valeur sacrée pour Calliclès, consiste en leur non-asservissement, il y a toutes les raisons de croire que l'éthique sera déduite de l'observation de la nature et non de la loi.

La liberté dans le domaine de la parole, la franchise (parrhesia), est également l'un des lieux d'enracinement du naturalisme de Calliclès. Sa parrhesia, mentionnée à quatre reprises et soulignant toujours par le fait même le radicalisme de ses thèses (487a-b, 491e, 492d, 521a), est un élément clef du dialogue puisqu'elle permet la critique des conventions ${ }^{60}$. Cet amour de la franchise amène d'abord Calliclès à constater que ni Gorgias ni Polos n'ont été pleinement convaincus par l'argumentation de Socrate, qui leur a tout simplement « clou[é] le bec » (482e) à cause du désir du premier de « se conformer aux règles de la société humaine » (482d) et de la honte du second de révéler le contenu exact de sa pensée. La parrhesia est un outil de premier choix pour révéler le caractère artificiel des nomoi et pour déterrer les désirs les plus profonds.

L'attachement à la parrhesia, commun à Diogène et à Calliclès, est en fait un excellent outil pour voir tout le fossé qui sépare leurs deux anticonventionnalismes. Calliclès, en effet, use de la parrhesia au début de son entretien avec Socrate, lorsqu'il affirme les deux pendants de son éthique naturaliste, mais dès qu'il est question de comparer son hédonisme avec la façon non conventionnelle de vivre la vie du kinaidos, il recule ${ }^{61}$. Il serait quelque peu fantaisiste de considérer que Calliclès croit en fait que le $\mathrm{ki}$ naidos a une vie digne, mais qu'il le cache, surtout au regard de ce que nous avons avancé sur les flottements de son hédonisme, hédonisme qui serait plus un rejet de toute forme de contrôle externe qu'une réelle vénération du plaisir ( $c f$. section I, 2. A.). On peut par ailleurs comprendre que Calliclès n'est anticonventionnaliste que concernant les questions de justice, mais non en ce qui a trait aux questions proprement culturelles c'est-à-dire aux mœurs sexuelles et alimentaires, à la piété et à la consommation matérielle. Tant s'en faut, donc, que Calliclès soit antiprométhéen comme Diogène (ce dernier s'essaie à manger de la viande crue dans deux anecdotes [D. L. VI $34 ; 76=S S R$ V B $93 ; 94]$ ), qu' il propose, comme lui, une sexualité qui ne s'embarrasse pas des conventions (D. L. VI $46=S S R$ V B 147) et qu'il dénonce certaines formes de piété (D. L. VI $37=S S R$ V B 344) et la consommation de biens inutiles ( $c f$. section I, B. 2).

60. Monoson 2000, p. 163.

61. Id. p. 164. 


\section{Diogène : se libérer de l'esclavagisme des désirs engendrés par la doxa}

On sait peu de choses sur le passé de Diogène avant qu'il n'ait été frappé par les épreuves et n'ait assimilé l'enseignement de la nature, sinon qu'il a été déçu des conventions (D. L. VI $20=S S R$ V B 2). La majorité des anecdotes que nous avons retenues sur lui manifestent son anticonventionnalisme : critique de la piété (D. L. VI $37=S S R$ V B 344; $42=S S R$ V B 344), masturbation en public (D. L. VI $46=S S R$ V B 147), réflexions sur la trop grande force des symboles (D. L. VI $35=S S R$ V B 276), rejet des institutions comme le mariage (D. L. VI $29=S S R$ V B 297), etc. Il semble raisonnable de croire que c'est en partie sur son anticonventionnalisme, sur la « falsification de la monnaie » (D. L. VI $20=S S R$ V B 2), que se fonde son entreprise de tirer une éthique de l'observation de la nature. Tout comme chez Calliclès, sa soif de liberté (D. L. VI $71=S S R$ V B 291) et son attachement à la parrhesia (D. L. VI $69=$ SSR V B 473) sont révélateurs de ce lien. De fait, l'amour de Diogène pour la liberté ne peut que l'amener, lorsqu'il est couplé à la ferme impression que les conventions et leurs conséquences la menacent, non seulement à rejeter ces dernières, mais à s'inspirer de ce qui s'y oppose diamétralement, la nature : Diogène le cynique « sacrifia les lois à la nature (D. L. VI $71=S S R$ V B 291) ». Les produits de la civilisation provoquent le malheur : l'amour de l'argent est la source de tous les maux (D. L. VI $50=S S R$ V B 228); l'économie de marché fait perdre le sens de la vraie valeur des choses (D. L. VI $35=S S R$ V B 276). Diogène croit à l'inutilité des solutions conventionnelles pour être heureux ; il dénonce la bêtise humaine, qu'il lie à la société puisqu'il oppose coutume et valeurs naturelles tout en associant ces dernières à la liberté d'Héraclès (D. L. VI 71 = SSR V B 291). Bref, la liberté de Diogène se vit dans le mépris de ce qui tient l'homme en esclavage, à savoir la convention. Contrairement à Calliclès, la liberté a pour Diogène un contenu positif: elle suppose de réaliser sa nature d'homme en contrôlant ses désirs.

Le corollaire de son mépris de la convention est un désir d'authenticité identifié dans la nature: ainsi s'efforce-t-il, en employant la parrhesia, de connaître les choses telles qu'elles sont profondément, y compris lui-mê$\mathrm{me}^{62}$. Dès lors que la civilisation est vue comme source d'artifices et de malheurs, la quête d'authenticité s'oriente vers la nature, au détriment de l'usage et des relations courtoises. À l'opposé de Calliclès, c'est donc non une généalogie de la morale qui est le socle du naturalisme de Diogène, mais une généalogie du malheur des gens (D. L. VI $44=S S R$ V B 322).

Manifestation de son appréciation de cette méthode, Diogène n'hésite pas, comme Calliclès, à utiliser l'argument d'origine : 
Il n'y a pas lieu d'être reconnaissants à nos parents de notre naissance, puisque c'est à la nature que ce qui est doit l'existence. [...] En effet, la génération est la conséquence directe de l'instinct sexuel ( $\dot{\eta} \gamma \dot{\alpha} \rho \gamma^{\dot{\varepsilon} \nu \varepsilon \sigma \iota \varsigma} \dot{\alpha} \phi \rho \circ \delta \sigma \sigma i \omega \nu$

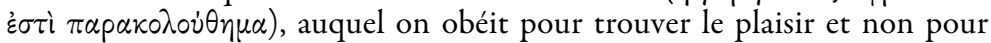

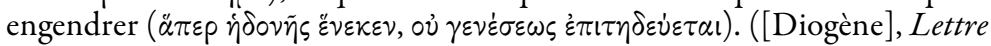
$21=\operatorname{SSR}$ V B 551.)

Autrement dit, le mérite qu'aurait un parent à enfanter est anéanti parce qu'il n'est que la conséquence de ses désirs. La recherche de l'origine naturelle trahit un rejet de la convention, rejet qui constitue un présupposé encourageant Diogène à déduire une éthique de l'observation de la nature.

\section{B. De l'anti-intellectualisme au naturalisme}

\section{Calliclés: rejeter l'elenchos et le mode de vie du philosophe}

L'anti-intellectualisme de Calliclès est aveuglant dans de nombreux passages du Gorgias: d'une part, dans sa critique hargneuse de la philosophie (484c-486d); d'autre part, durant tout le dialogue, dans sa façon de répondre à Socrate. Cet «anti-intellectualisme ambiant» se manifeste d'abord par le refus de Calliclès d'entrer en dialogue avec Socrate et par la mauvaise foi qu'il déploie pour y mettre un terme (497a ; 487b-c; 498c-d ; 499b ; 501c ; 505c ; 506c ; 507a ; 510a ; 513d ; 514a ; 516b-d). Ensuite, on le remarque dans le refus d'admettre les méthodes logiques de Socrate, qui attache une grande importance aux mots utilisés, aux définitions, aux contre-exemples. Calliclès accuse son interlocuteur d'utiliser des « chevilles d'orateur populaire » (482e), « des trucs $\gg(483 \mathrm{a})$ et des «tours de sophistes » (497a); de «faire la chasse aux mots» (489b); de parler de «vivres, de boissons, de médecins - des bêtises » (490c); et de «retourner sens dessus dessous tout ce qu'on a dit » (511a). Toute tentative de Socrate de s'éloigner de l'évidence supposée des sens et de l'opinion, de trouver les conditions nécessaires et suffisantes de quelque chose et de procéder à des analogies est d'emblée rejetée par Calliclès.

Cet anti-intellectualisme est non seulement enraciné dans le dialogue, mais dans les deux pendants de la thèse éthique et naturaliste de Calliclès, la domination des plus forts et l'absence hédoniste de contraintes. D'abord, comme le naturalisme de Calliclès l'amène à postuler une forme d'hédonisme où les passions ne doivent jamais être soumises à quoi que ce soit, même à la raison (492a), et où tout doit, chez un homme, participer à l'accomplissement et à l'entretien de ses désirs, la seule idée d'une raison qui agit seulement pour elle-même paraît méprisable. Inversement, puisque la raison pratiquée pour elle-même repousse Calliclès au point où « quand [il se] trouve en face d'hommes qui philosophaillent, [il] éprouve exactement le même sentiment qu'en face de gens qui babillent et qui s'expriment comme des enfants » (485b), il croit que «c'est indigne d'un homme et cela 
mérite des coups » (485c), il est compréhensible qu'il s'inscrive en faux contre le modèle de vie philosophique et qu'il se tourne vers la nature.

Son anti-intellectualisme est donc une position concomitante à la priorité épistémique qu'il accorde aux sens pour la découverte d'une éthique, priorité qui contredit Socrate, lorsque celui-ci affirme qu'il est impossible d'être aveugle aux bienfaits de la justice et au malheur qu'apporte une âme injuste (479b). En effet, dès lors que quelqu'un accorde que c'est l'observation qui lui révèle s'il est heureux ou non, il ne peut admettre une telle thèse.

De surcroît, une observation initiale de la nature fait voir à Calliclès le peu de valeur de la philosophie, qu'il pratiquait quand il était jeune et dont il est déçu (487c). Il remarque que les puissants sont bien sûr dotés d'une intelligence pratique, mais qu'ils ne s'adonnent pas à la philosophie, une fois âgés. Le philosophe, lui, passe sa vie «à chuchoter dans son coin avec trois ou quatre jeunes gens, sans jamais proférer la moindre parole libre, décisive, efficace » (485d-e). L'absence d'efficacité de la philosophie ne réside pas tant dans un échec épistémique, c'est-à-dire par exemple dans l'absence d'une théorie qui rendrait réellement compte de la nature du monde, mais dans un échec pratique. D'abord, le philosophe n'a pas les outils pour se défendre de l'injustice (485b) et il est en ce sens un esclave (483b ; 485b), dont « la parole n'est pas libre » (485e). C'est la raison pour laquelle Calliclès met Socrate en garde et lui suggère d'être flatteur avec le peuple (521b). Ensuite, le philosophe est un sous-homme puisque, dépourvu de l'estime de la cité (486c), il est éloigné des débats publics « là où les hommes se rendent remarquables » (485d) et manifeste par là son inaptitude au courage (486a). Pire, la philosophie fait oublier la façon de vivre des hommes (484c-d), leurs plaisirs et leurs lois - Calliclès, même s'il rejette la loi croit qu'il faut la maîtriser pour ne pas subir l'injustice. Conséquemment, le philosophe se rend ridicule, il est «pervert [i] sous une apparence puérile » (485d). Enfin, les arguments logiques de la philosophie font piètre figure à côté d'arguments psychologiques, laisse croire Calliclès en refusant la réfutation socratique $(513 \mathrm{c})^{63}$. Bref, après avoir développé un anti-intellectualisme à partir de la simple observation d'une partie de la nature, à savoir les relations de pouvoir des hommes dans la cité, Calliclès est poussé à donner une valeur normative à ses observations, par refus des conséquences de la philosophie.

\section{Diogène : repousser le savoir pour lui-même sans mépriser la raison}

Il est intéressant de remarquer que Diogène aurait pu se réapproprier la critique de Calliclès à l'endroit de Socrate : le cynique ne s'illustre pas dans

63. Canto 2007, p. 352, n. 210. 
la cité qu'il se met à dos, mais il ne vit pas dans l'ombre et ne se suffit pas de la présence réconfortante de ses disciples et de ses livres (D. L. VI 104 = SSR V A 369); au contraire, il vit en permanence dans l'espace public et exerce son courage à chaque instant. Il ne faut donc pas s'étonner que les liens entre l'anti-intellectualisme de Diogène (D. L. VI 39; 103-104 = SSR V B 479, 371) et son naturalisme soient similaires à ceux qu'on trouvait chez Calliclès. L'anti-intellectualisme de Diogène se constitue en effet d'une part d'un mépris, comme chez Calliclès, de la philosophie traditionnelle et des écoles (D. L. VI 24; $40=\operatorname{SSR}$ V B $487 ; 63$ ), mais aussi des livres (D. L. VI $48=S S R$ V B 118) et des sciences (D. L. VI $73=S S R$ V B $370)^{64}$; d'autre part, d'une vision pragmatique de la philosophie (D. L. VI $63=S S R$ V B 360) et de la conviction qu'il s'agit de la seule façon d'être heureux ${ }^{65}$. De fait, « [1]e désir de connaître un objet pour lui-même et de mener une enquête théorique n'est visiblement pas considéré par les cyniques comme un besoin naturel et donc légitime $\gg^{66}$. L'anti-intellectualisme et le naturalisme de Diogène se renforcent l'un l'autre : à force de regarder la nature, Diogène méprise l'intellectuel ; plus il est attentif aux agissements de ce dernier, plus il se tourne vers la nature ${ }^{67}$. Autrement dit, la pratique d'une philosophie centrée sur l'homme exacerbe la sensibilité de Diogène aux incohérences de ses concitoyens et à l'ineptie de leurs discours :

Il trouvait étrange que les grammairiens fassent tant de recherches sur les défauts d'Ulysse tout en ignorant leur propre malice. Et il s'étonnait aussi de voir [...] les mathématiciens fixer leurs regards sur le soleil et la lune, mais ne pas remarquer ce qui se passe à leurs pieds; les orateurs mettre tout leur zèle à parler de la justice, mais ne point du tout la pratiquer, et encore les philosophes blâmer l'argent, mais le chérir par-dessus tout. (D. L. VI 27 $28=$ SSR V B 374.)

L'attribution initiale d'une valeur normative à la nature, aux premiers moments de cette dynamique de renforcement entre l'anti-intellectualisme et le naturalisme, semble naître d'un dégoût des affaires humaines.

Par ailleurs, pour accepter que la nature soit un modèle, il faut avoir la certitude que ce qui fait le bonheur de l'animal fait aussi celui de l'homme. En effet, si Diogène accepte de suivre le modèle d'une souris, c'est soit qu'il est à court de modèle (ce qui est faux : il a les dieux), soit qu'il croit profondément en la ressemblance de l'homme et de l'animal. Cette dernière thèse est endossée par Diogène : « la distinction entre le corps et l'âme

64. Meilland 1983, p. 233-234.

65. Husson 2011, p. 68-69.

66. Husson 2011, p. 64.

67. Cela évoque les paroles de Bismarck: «plus j'apprends à connaître l'homme, plus j'aime mes chiens. » 
n'[a] guère joué de rôle dans la réflexion de Diogène sur l'ascèse. Pour ce dernier, en fait, c'est tout l'être humain qui globalement s'entraîne à la vertu quand il affronte le froid, le chaud, la soif, la faim ou la maladie ${ }^{68}$. $\gg$ Cela, il l'accepte d'emblée, d'une manière sinon anti-intellecutaliste, du moins non intellectuelle, non pas parce que le matérialisme est intellectuellement moins riche que la théorie des idées ou une forme de dualisme des substances, mais parce que Diogène n'élabore aucun discours métaphysique sur l'identité personnelle. Il se situe « en deçà du débat lui-mê$\mathrm{me}^{69}$ 》 puisqu'il a choisi l'ascèse comme solution aux problèmes du monde. Ce faisant, il «falsifie » la philosophie elle-même ${ }^{70}$. Bref, en vertu d'un anti-intellectualisme latent qui le mène à ne pas se lancer dans des investigations sur la nature de l'homme - il cherche des hommes, pas ce qu'est l'homme -, Diogène peut accepter « les leçons de la nature » sans scepticisme, par un acte de foi ${ }^{71}$. Cette impatience à l'égard de la recherche théorique, particulièrement sur la définition de l'homme, impatience manifeste dans le type de voie qu'il emprunte vers le bonheur, à savoir la voie courte, ne pourrait être mieux exprimée que par cette anecdote :

Platon avait défini l'homme «un animal bipède, et sans plumes », et on l'applaudissait; Diogène pluma alors un coq et l'apporta à la salle de cours en s'écriant: « Voici l'homme de Platon! » On ajouta donc à la définition : « muni de larges ongles ». (D. L. VI $40=\operatorname{SSR}$ V B 63.)

En somme, en rejetant toute l'entreprise définitionnelle et en acceptant les ponoi comme mode d'acquisition de la connaissance ${ }^{72}$, en accordant, comme Calliclès, une priorité épistémique à ce qu'il voit et ressent, Diogène se destine dès le départ à accepter les animaux comme modèles.

\section{Conclusion}

Ont d'abord été mises en parallèle deux éthiques: celle de la domination des hommes les plus forts, les plus sagaces et les plus courageux au sens où ils n'ont pas peur de s'investir dans la vie politique, des hommes qui ne se laissent pas non plus dominer par la tempérance, mais qui laissent libre cours à leurs passions ; celle de la maîtrise des circonstances, par tous, en renouant avec la facilité de la nature et en pratiquant une ascèse qui prescrit la limitation des besoins, l'augmentation de la résistance et l'affranchissement des désirs. Devant les étonnantes contradictions qu'elles

68. Goulet-Cazé 1986, p. 212.

69. Husson 2011, p. 54.

70. Goulet-Cazé 1986, p. 15-16.

71. Husson 2011, p. 71.

72. Husson 2011, p. 64. 
présentaient, les natures desquelles elles s'inspirent ont été visitées : celle qui inclut les cités et les hommes et dont les modèles ne sont jamais les animaux, mais toujours les tyrans, dans leurs rapports à ceux qu'ils dominent ; celle qui exclut en général les hommes parce qu'ils sont trop éloignés de leur nature, mais inclut les enfants et les petits animaux en prenant leur relation à eux-mêmes comme modèles. Devant le risque persistant d'oublier que toute observation de la nature est porteuse de présupposés théoriques ou de positions philosophiques préalables, plusieurs de celles-ci ont été comparées chez Calliclès et Diogène : chez le premier, un anticonventionnalisme fondé sur une généalogie de la morale et une conception de la liberté comme absence de contrainte extérieure et un anti-intellectualisme profondément enraciné dans une critique de la philosophie, accusée de faire encourir dangers et humiliations; chez le second, un anticonventionnalisme érigé à partir d'une brève généalogie de l'origine des malheurs et d'une conception positive de la liberté, comprise comme suprématie de la raison pratique, et un anti-intellectualisme vécu par l'ascèse et les ponoi et qui incite à suivre les conseils de la nature, fussent-ils ceux d'une souris. Il n'y a donc plus à s'étonner que les éthiques de Diogène et de Calliclès divergent à ce point. Il y a plutôt à s'interroger sur la pertinence de l'entreprise naturaliste et surtout sur la possibilité de déterminer une notion acceptable et commune de nature. Faute de cela, l'autorité de la nature pourra toujours être contestée par celle d'une autre nature ${ }^{73}$.

Il peut être intéressant, une fois le parallèle établi entre les deux pensées, de se demander comment Socrate aurait pu réfuter Diogène ${ }^{74}$. S'il avait

73. Je remercie John Thorp pour son commentaire de ce passage lors du Deuxième Colloque Canadien de Philosophie Ancienne, dans lequel il rappelle qu'il est peut-être plus laborieux de définir ce qu'est la nature que d'échafauder une éthique, ce qui aurait pour effet de miner l'entreprise naturaliste.

74. Nous faisons nôtre la position très répandue suivant laquelle le véritable fondateur du cynisme serait Diogène, et non pas Antisthène, de sorte qu'il n'y aurait pas de filiation entre le socratisme et le cynisme à travers la figure d'Antisthène. Cette prétendue filiation serait plutôt le fait d'une invention doxographique visant à redorer les origines de la Stoa en reliant son fondateur (Zénon) à Socrate en passant successivement par Antisthène, Diogène et Cratès ( $c f$. déjà Dudley 1937, p. 4). Notre article n'avait pas pour objectif de prendre position sur cette question, qui demeure par ailleurs controversée, comme le montre l'article récent de Fuentez González 2013. Néanmoins, des indices supplémentaires mis en lumière par notre article peuvent apporter de l'eau au moulin des tenants de l'absence de filiation : notre analyse révèle en effet que les positions de Diogène et de Socrate sur la possibilité de déduire une éthique de l'étude de la nature sont diamétralement opposées. Plus particulièrement, alors que Diogène affectionne le modèle animal comme inspiration concrète pour l'atteinte du bonheur, Socrate n'y fait jamais appel en ce sens. Dans la République, par exemple, l'utilisation de la figure du chien comme bon gardien vise ou bien à créer un effet ironique ou métaphorique ou encore, et plus probablement, à s'inspirer de ce qu'il y a de 
employé une méthode similaire à celle que Rachel Barney lui attribue c'est-à-dire ne pas attaquer le postulat voulant qu'on puisse tirer quelque leçon que ce soit de la nature -, on peut croire qu'il aurait cette fois non pas critiqué la conception que Diogène se fait de la nature - après tout, il s'agit de la même nature abondante que le Socrate de Xénophon décrit dans les Mémorables -, mais celle de la divinité et de l'homme. Vouloir se rapprocher de sa nature et de celle des dieux sans exercer sa raison paraît dangereux et illusoire parce que l'homme parfait n'est justement pas une pierre comme le dit si bien Calliclès; c'est un être dont la raison théorique devrait s'exercer.

plus civilisé, domestiqué, chez l'animal ( $c f$. Hotes 2014, qui montre justement le fossé qu'il y a entre le chien de Diogène et celui de Platon).

Je tiens à remercier Louis-André Dorion (Université de Montréal) pour ses très précieux commentaires sur différentes versions de cet article. 


\section{BIBLIOGRAPHIE}

ADAMS, J.-L. 1945 : « The Law of Nature in Greco-Roman Thought », The Journal of Religion, 25 (1945/2), p. 97-118.

BARNEY, R. 2011 : «Callicles and Thrasymachus », dans Stanford Encyclopedia of Philosophy, URL : http://plato.stanford.edu/entries/callicles-thrasymachus/.

Berman, S. 1991 : « Socrates and Callicles on Pleasure », Phronesis, 36/2, p. 117-140.

BRANCACCI, A. 1993 : «Érotique et théorie du plaisir chez Antisthène », dans M.-O. Goulet-Cazé \& R. Goulet (éd.), Le Cynisme ancien et ses prolongements. Actes du Colloque international du CNRS (Paris, 22-25 juillet 1991), Paris, 1993, p. 35-55.

CANTO-Sperber, M. 2007 (trad.) : Platon, Gorgias, traduction inédite, introduction et notes, nouv. éd. revue et augmentée, Paris, 2007 (coll. GF 1326).

Chevarie-CossetTe, S.P. 2013 : «Deux formes de naturalisme antique: la nature comme fondement d'éthiques contradictoires », dans É. Litalien et al., Peut-on tirer une éthique de létude de la nature?, Montréal, 2013, p. 103-112 (Les Cahiers d'Ithaque).

Cross, R. C. 1950 : «Virtue and Nature », Proceedings of the Aristotelian Society, N. S. 50 (1949-1950), p. 123-137.

DodDs, E. R. 1959 (éd.) : Plato, Gorgias, A Revised Text with Introduction and Commentary, Oxford, 1959.

DORION, L. A. 2000 (trad.) : Xénophon, Mémorables, tome I : Introduction générale, Livre I, Paris, 2000 (Collection des Universités de France).

Dudley, D. R. 1937 : A History of Cynicism from Diogenes to the 6th cent. A. D., Londres, 1937.

FlORES-JUNIOR, O. 2005 : «Cratès, la fourmi et l'escarbot : les cyniques et l'exemple animal », Philosophie antique, 5 (2005), p. 135-171.

Fuentes GONZÁlez, P. P. 1998 : Les diatribes de Télès, Introduction, texte revu et commentaire des fragments, avec en appendice une traduction espagnole, Paris, 1998 (Histoire des doctrines de l'antiquité classique, 23).

-2003 : «Necesitaban de un amigo los cínicos antiguos? », Bitarte, 31 (2003/10), p. 5172.

- 2013: «En defensa del encuentro entre dos Perros, Antístenes y Diógenes : historia de una tensa amistad », Cuadernos de Filología Clásica: Estudios Griegos e Indoeuropeos, 23 (2013), p. 225-267.

GOUlET-CAZÉ M.-O. 1986: L'Ascèse cynique : un commentaire de Diogène Laërce VI 70-71, Paris, 1986 (Histoire des doctrines de l'antiquité classique, 10).

- 1992 : «Avant-propos: Les Cyniques et la "falsification de la monnaie" », dans Paquet 1992 , p. 5-29.

HADOT, P. 1995 : Qu'est-ce que la philosophie antique?, Paris, 1995 (Folio-Essais).

Hotes, M. 2014: «Du chien au philosophe: l'analogie du chien chez Diogène et Platon », Revue de Philosophie Ancienne, 32/1 (2014), p. 3-33.

Husson, S. 2011 : La République de Diogène: une cité en quête de la nature, Paris, 2011 (Histoire des doctrines de l'antiquité classique, 40).

Judet DE LA Combe, P. 1982 : Agamemnon 2, dans J. Bollack et P. Judet de la Combe, L'Agamemnon d'Eschyle: le texte et ses interprétations, 3 vol., Lille, 1981-1982 (Cahiers de Philologie, 8).

Kalouche, F. 2003: «The Cynic Way of Living », Ancient Philosophy, 23/1 (2003), p. 181-194. 
LONG A. A. et D. N. SEDley 1997 (éd.) : Les Philosophes hellénistiques (trad. J. Brunschwig et P. Pellegrin), 3 vol., Paris, 2001 (GF 641-643).

MeILland, J.-M. 1983 : «L'anti-intellectualisme de Diogène le cynique », Revue de théologie et de philosophie, 115 (1983), p. 233-246.

Monoson, S. 2000 : Plato's Democratic Entanglements: Athenian Politics and the Practice of Philosophy, Princeton, 2000.

PAQUeT, L. 1992 : Les Cyniques Grecs: Fragments et témoignages, choix, traduction, introduction et notes, Paris, 1992 (Classiques de la philosophie).

STAuffer, D. 2002, « Socrates and Callicles: A Reading of Plato's "Gorgias" », The Review of Politics, 64 (2002), p. 627-658.

Voelke, A.-J. 1982, «Droit de la nature et nature du droit: Calliclès, Épicure, Carnéade », Revue philosophique de la France et de l'Étranger, 172 (1982), p. 267-275. 